\title{
Metabolic flux analysis of wild-type Escherichia coli and mutants deficient in pyruvate-dissimilating enzymes during the fermentative metabolism of glucuronate
}

Correspondence

Ramon Gonzalez

Ramon.Gonzalez@rice.edu

Received 9 November 2009

Revised 15 February 2010 Accepted 16 February 2010

\author{
Abhishek Murarka, ${ }^{1}$ James M. Clomburg ${ }^{1}$ and Ramon Gonzalez ${ }^{1,2}$ \\ 1'Department of Chemical and Biomolecular Engineering, Rice University, Houston, TX, USA \\ ${ }^{2}$ Department of Bioengineering, Rice University, Houston, TX, USA
}

The fermentative metabolism of D-glucuronic acid (glucuronate) in Escherichia coli was investigated with emphasis on the dissimilation of pyruvate via pyruvate formate-lyase (PFL) and pyruvate dehydrogenase (PDH). In silico and in vivo metabolic flux analysis (MFA) revealed that PFL and PDH share the dissimilation of pyruvate in wild-type MG1655. Surprisingly, it was found that PDH supports fermentative growth on glucuronate in the absence of PFL. The PDHdeficient strain $(\mathrm{Pdh}-)$ exhibited a slower transition into the exponential phase and a decrease in specific rates of growth and glucuronate utilization. Moreover, a significant redistribution of metabolic fluxes was found in PDH- and PFL-deficient strains. Since no role had been proposed for $\mathrm{PDH}$ in the fermentative metabolism of $E$. coli, the metabolic differences between MG1655 and $\mathrm{Pdh}-$ were further investigated. An increase in the oxidative pentose phosphate pathway (ox-PPP) flux was observed in response to PDH deficiency. A comparison of the ox-PPP and $\mathrm{PDH}$ pathways led to the hypothesis that the role of $\mathrm{PDH}$ is the supply of reducing equivalents. The finding that a $\mathrm{PDH}$ deficiency lowers the $\mathrm{NADH}: \mathrm{NAD}^{+}$ratio supported the proposed role of $\mathrm{PDH}$. Moreover, the $\mathrm{NADH}: \mathrm{NAD}^{+}$ratio in a strain deficient in both $\mathrm{PDH}$ and the ox-PPP (Pdh-Zwf-) was even lower than that observed for Pdh-. Strain Pdh-Zwf- also exhibited a slower transition into the exponential phase and a lower growth rate than Pdh-. Finally, a transhydrogenase-deficient strain grew more slowly than wild-type but did not show the slower transition into the exponential phase characteristic of Pdh- mutants. It is proposed that PDH fulfils two metabolic functions. First, by creating the appropriate internal redox state (i.e. appropriate $\mathrm{NADH}: \mathrm{NAD}^{+}$ratio), $\mathrm{PDH}$ ensures the functioning of the glucuronate utilization pathway. Secondly, the NADH generated by PDH can be converted to NADPH by the action of transhydrogenases, thus serving as biosynthetic reducing power in the synthesis of building blocks and macromolecules.
Abbreviations: AcCoA, acetyl-CoA; ACK, acetate kinase; MFA, metabolic flux analysis; non-ox-PPP, non-oxidative pentose phosphate pathway; ox-PPP, oxidative pentose phosphate pathway; $\mathrm{PDH}$, pyruvate dehydrogenase; PFL, pyruvate formate-lyase; PP, pentose phosphate; PPP, pentose phosphate pathway; PTA, phosphotransacetylase; TCA cycle, tricarboxylic acid cycle.

A reaction network model for glucuronate fermentation by Escherichia coli, list of metabolites and results of the in vivo metabolic flux analysis of wild-type E. coli MG1655 and mutants deficient in the pyruvatedissimilating enzymes pyruvate dehydrogenase and pyruvate formatelyase, and in silico metabolic flux analysis of wild-type E. coli and mutants deficient in pyruvate dehydrogenase, pyruvate-formate lyase, glucose-6phosphate dehydrogenase and phosphotransacetylase enzymes, are available as supplementary information with the online version of this paper.

\section{INTRODUCTION}

D-Glucuronic acid (glucuronate) is a naturally occurring six-carbon sugar acid found in both plants and animals, the fermentative metabolism of which is of importance for fundamental and applied research. For example, glucuronic and 4-O-methyl-glucuronic acids are major side chains of xylans present in most plants considered as feedstocks for the production of biofuels (Lawford \& Rousseau, 1997), an application in which Escherichia coli is one of the preferred organisms (Atsumi \& Liao, 2008). Glucuronate is also found in the intestine of mammals, where E. coli is the predominant facultative anaerobe (Finegold et al., 1983), and its metabolism plays an important role in the colonization of the large intestine by this bacterium (Peekhaus \& Conway, 1998). 
E. coli utilizes glucuronate as the sole carbon and energy source under fermentative conditions using the pathways summarized in Fig. 1 (Mandrand-Berthelot et al., 2004). Once inside the cells, glucuronate is isomerized to $\mathrm{D}$-fructuronate by D-glucuronate isomerase $(u x a C)$. D-Fructuronate then undergoes an $\mathrm{NADH}$-dependent reduction to D-mannonate by D-mannonate oxidoreductase $(u x u B)$. D-mannonate dehydratase $(u x u A)$ subsequently catalyses dehydration to yield 2-dehydro-3-deoxy-D-gluconate (KDG). At this point, 2-keto-3-deoxygluconokinase $(k d g K)$ phosphorylates KDG to yield 2-dehydro-3-deoxy-Dgluconate-6-phosphate (KDPG), which is an intermediate in the Entner-Doudoroff pathway. KDPG is further cleaved by 2-keto-3-deoxygluconate-6-phosphate aldolase (eda) to produce the glycolytic intermediates pyruvate and glyceraldehyde 3-phosphate (Mandrand-Berthelot et al., 2004). These two intermediates are processed through common glycolytic, gluconeogenic and fermentative pathways, and reductive and oxidative branches of the tricarboxylic acid (TCA) cycle, to generate precursor metabolites, ATP, and reducing power for biomass synthesis along with several fermentation products (Fig. 1). Pyruvate also serves as the main switching point between respiratory and fermentative metabolism. In this context, pyruvate formate-lyase (PFL) and pyruvate dehydrogenase (PDH) are considered the major routes for pyruvate dissimilation in E. coli (Sawers \& Clark, 2004). During aerobic growth, pyruvate is converted to acetyl-CoA (AcCoA), carbon dioxide and NADH by PDH. For anaerobic growth, on the other hand, PFL replaces PDH in the formation of AcCoA, and generates formate as co-product.

The work reported here studied the fermentative metabolism of glucuronate in E. coli with emphasis on pyruvate dissimilation. It was found that $\mathrm{PDH}$ can support fermentative growth on glucuronate in the absence of PFL. In silico and in vivo metabolic flux analyses (MFA) were used to characterize glucuronate fermentation in the wild-type and in recombinant strains lacking PDH and PFL. Changes in metabolic fluxes brought about by the $\mathrm{PDH}$ and PFL deficiencies provided the basis to hypothesize that the role of PDH during glucuronate fermentation is to provide reducing equivalents for glucuronate utilization and biosynthesis.

\section{METHODS}

Strains, plasmids and genetic methods. Strains, plasmids and primers used in this study are listed in Table 1. K12 strain MG1655, used as the wild-type strain, and mutants $\mathrm{Pdh}-$ (PDH-deficient) and Pfl- (PFL-deficient) were obtained from the University of Wisconsin E. coli Genome Project (http://www.genome.wisc.edu: Kang et al., 2004). Single-gene deletion mutants JW1841 ( $\Delta z w f-F R T-K m-F R T)$,

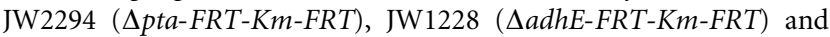
JW1375 ( $\Delta l d h A-F R T-K m-F R T)$ were obtained from the National BioResource Project, National Institute of Genetics, Japan (Baba et al., 2006). Double mutant SthA-PntA- (SthA-, soluble pyridine nucleotide transhydrogenase-deficient; PntA-, membrane-bound pyridine nucleotide transhydrogenase-deficient) was obtained from the laboratory of Dr U. Sauer, Institute of Molecular Systems Biology, Zurich, Switzerland (Sauer et al., 2004). Single mutants Zwf(glucose-6-phosphate dehydrogenase-deficient), Pta- (phosphotransacetylase-deficient), Ldh- (lactate dehydrogenase-deficient) and Adh - (alcohol/acetaldehyde dehydrogenase-deficient) were constructed by introducing the $z w f, p t a, l d h A$ and $a d h E$ deletions from JW1841, JW2294, JW11228 and JW1375, respectively, into MG1655 using P1 phage transduction. Double mutant $\mathrm{Pdh}-\mathrm{Zwf}-$ was constructed by transferring the aceF mutation from strain Pdhinto strain $\mathrm{Zwf}-$. Double mutant $\mathrm{Pfl}-\mathrm{Ldh}-$ was obtained by transferring the pflB mutation from strain Pfl- into strain Ldh-. Finally, double mutant $\mathrm{Pta}-\mathrm{Adh}-$ was constructed by transferring the adhE mutation from strain JW1228 into strain Pta-. Details of the specific protocol used in phage transduction are available elsewhere (Yazdani \& Gonzalez, 2008; Miller, 1972).

Manufacturers' protocols and standard methods (Miller, 1972; Sambrook et al., 1989) were followed for DNA purification (Qiagen), restriction endonuclease digestion (New England Biolabs) and DNA amplification (Stratagene and Invitrogen). The strains were kept in $32.5 \%, \mathrm{v} / \mathrm{v}$, glycerol stocks at $-80{ }^{\circ} \mathrm{C}$. Plates were prepared using Luria-Bertani (LB) medium containing $1.5 \%(\mathrm{w} / \mathrm{v})$ agar, and chloramphenicol and kanamycin were included (when required) at final concentrations of 34 and $50 \mu \mathrm{g} \mathrm{ml}^{-1}$, respectively.

Culture medium and cultivation conditions. The minimal medium designed by Neidhardt et al. (1974) supplemented with $5 \mathrm{~g}$ glucuronate $1^{-1}$ was used unless otherwise specified. A $10 \times$ concentrate of this medium was prepared and filter-sterilized before storing at $-20{ }^{\circ} \mathrm{C}$. Chloramphenicol and kanamycin were included when required at final concentrations of 34 and $50 \mu \mathrm{g} \mathrm{ml} \mathrm{m}^{-1}$, respectively. Chemicals were obtained from Fisher Scientific and Sigma-Aldrich.

Fermentations were conducted in a SixFors multi-fermentation system (Infors) with six $400 \mathrm{ml}$ working volume vessels and independent control of temperature $\left(37^{\circ} \mathrm{C}\right), \mathrm{pH}(7.4)$ and stirrer speed (300 r.p.m.) (Dharmadi et al., 2006). The system was fully equipped and computer controlled using the manufacturer's IRIS NT software. Each vessel was fitted with a condenser operated with a $4{ }^{\circ} \mathrm{C}$ cooling methanol-water supply to minimize evaporation. Anaerobic conditions were maintained as follows. The medium was autoclaved and sparged with ultra-high-purity argon (Matheson, TriGas), which was passed through an oxygen trap (Alltech Associates) to eliminate traces of oxygen. The vessels were then closed to prevent any exchange of gases. $\mathrm{NaOH}$ and $\mathrm{H}_{2} \mathrm{SO}_{4}$ (both $3 \mathrm{M}$ ) were used to control the $\mathrm{pH}$.

Pre-cultures used to inoculate the above fermenters were prepared as follows. A single colony was used to inoculate $17 \mathrm{ml}$ Hungate tubes (Bellco Glass) completely filled with MOPS media ( $\mathrm{pH}$ 7.4) supplemented with $5 \mathrm{~g}$ glucuronate $\mathrm{l}^{-1}, 10 \mathrm{~g}$ tryptone $\mathrm{l}^{-1}$ and $5 \mathrm{~g}$ yeast extract $1^{-1}$. The tubes were placed in a rotator and kept at $37^{\circ} \mathrm{C}$. Upon reaching a cell density between $\mathrm{OD}_{550} 0.3$ and 0.4 (measured using a BioMate 5 spectrophotometer, Thermo Scientific), the cultures were centrifuged and the pellet washed and resuspended in minimal medium ( $\mathrm{pH} 7.4$ ) to $\mathrm{OD}_{550} 0.02$. This inoculated minimal medium was incubated in completely filled Hungate tubes as described above until the cultures reached an $\mathrm{OD}_{550}$ between 0.3 and 0.4. An appropriate volume of the culture was harvested, the pellet resuspended in $10 \mathrm{ml}$ minimal medium, and the resulting suspension used to inoculate the fermenters with a target starting $\mathrm{OD}_{550}$ of 0.05 . Some experiments were conducted in partially filled tubes $(10 \mathrm{ml}$ medium) with an argon atmosphere. For this purpose, the medium was dispensed to the tubes and sparged with ultra-highpurity argon as described above for the fermentation vessels. These tubes were then inoculated with a preculture prepared as described above using $17 \mathrm{ml}$ Hungate tubes (Bellco Glass) completely filled with 


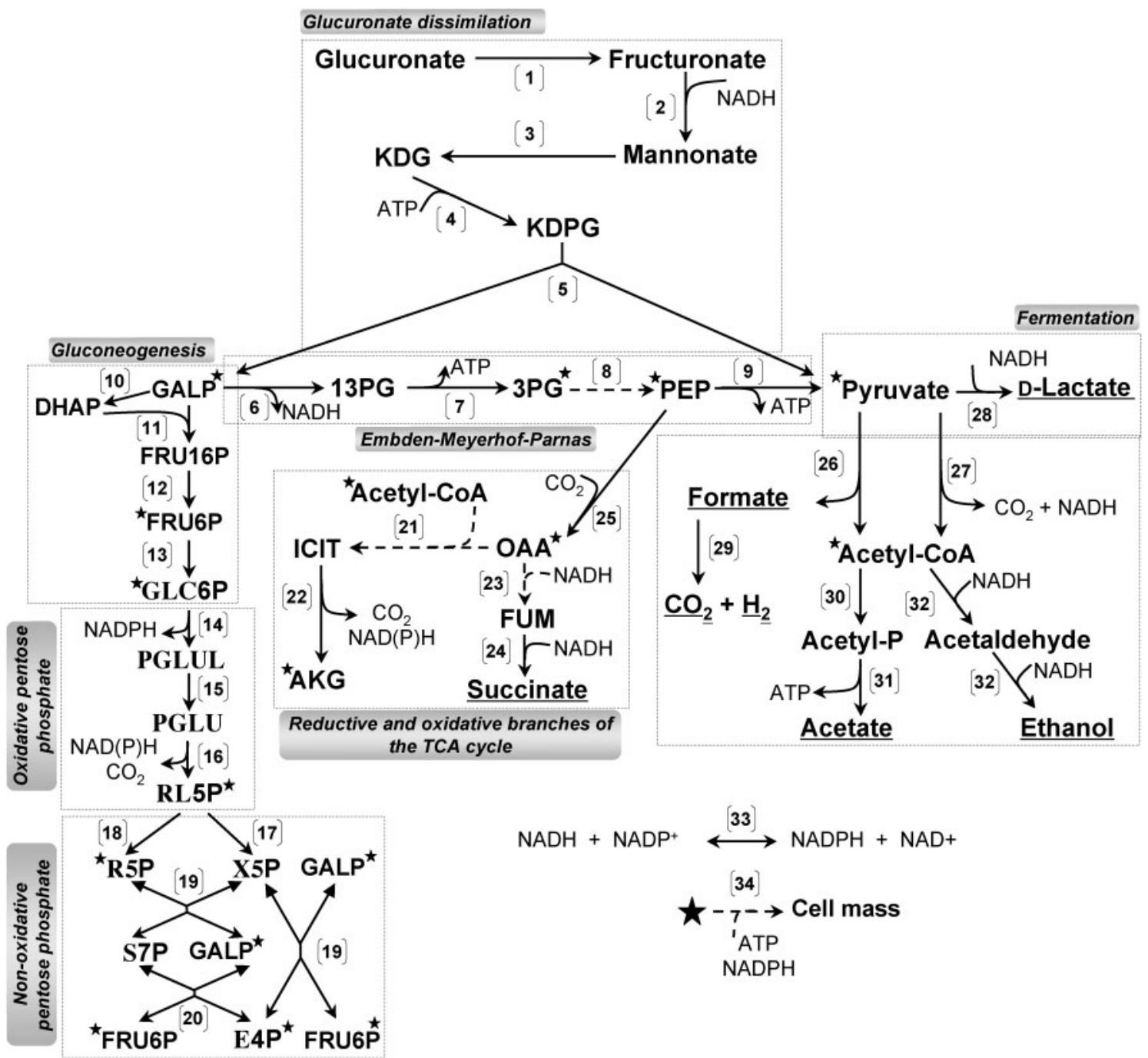

Fig. 1. Key metabolic pathways involved in the fermentative metabolism of glucuronate by $E$. coli. Glucuronate utilization: [1], D-glucuronate isomerase (uxaC); [2], D-mannonate oxidoreductase (uxuB); [3], D-mannonate dehydratase (uxuA); [4], 2-keto-3deoxygluconokinase (kdgK); [5], 2-keto-3-deoxygluconate-6-phosphate aldolase (eda). Embden-Meyerhof-Parnas: [6], glyceraldehyde-3-phosphate dehydrogenase (gapA); [7], phosphoglycerate kinase (pgk); [8], phosphoglycerate mutase $(g p m A)$ and enolase (eno); [9], pyruvate kinase I/ll (pykF/pykA). Gluconeogenesis: [10], triose phosphate isomerase (tpiA); [11], fructose bisphosphate aldolase class II (fbaA); [12], fructose-1,6-bisphosphatase I (fbp); [13], phosphoglucose isomerase (pgi). ox-PPP: [14], glucose-6-phosphate dehydrogenase (zwf); [15], 6-phosphogluconolactonase (pgl); [16], 6-phosphogluconate dehydrogenase (gnd). Non-ox-PPP: [17], ribulose phosphate 3-epimerase (rpe); [18], ribose-5-phosphate isomerases A (rpiA) and $\mathrm{B}($ rpiB); [19], transketolases I $(t k t A)$ and II $(t k t B) ;[20]$, transaldolases $\mathrm{A}(\operatorname{tal} A)$ and $\mathrm{B}($ talB). Oxidative and reductive branches of the TCA cycle: [21], citrate synthase ( $g / t A)$ and aconitases (acnA and $a c n B)$; [22], isocitrate dehydrogenase (icd); [23], malate dehydrogenase $(m d h)$ and fumarase isozymes $A(f u m A)$ and $B(f u m B)$; [24], fumarate reductase (frdABCD). Anaplerotic reaction: [25], phosphoenolpyruvate carboxylase ( $p p c)$. Pyruvate dissimilation: [26], pyruvate formate-lyase (pflB); [27], pyruvate dehydrogenase complex (aceEF-lpdA). Fermentation: [28], lactate dehydrogenase (IdhA); [29], formate hydrogen lyase $(f d h F, h y c B-F)$; [30], phosphotransacetylase (pta); [31], acetate kinase (ackA); [32], alcohol/acetaldehyde dehydrogenase $(a d h E)$. Transhydrogenases: [33], soluble (sthA) and membrane-bound ( $p n t A B$ ) pyridine nucleotide transhydrogenases. Cell growth: [34], synthesis of cell mass from precursor metabolites ( $\star$ ), ATP and reducing equivalents. Broken lines indicate multiple steps. Abbreviations: Acetyl-P, acetyl phosphate; AKG, $\alpha$-ketoglutarate; DHAP, dihydroxyacetone phosphate; E4P, erythrose 4-phosphate; FRU16P, fructose 1,6-bisphosphate; FRU6P, fructose 6-phosphate; FUM, fumarate; GALP, glyceraldehyde 3-phosphate; GLC6P; glucose 6-phosphate; ICIT, isocitrate; KDG, 2-dehydro-3-deoxy-D-gluconate; KDPG, 2-dehydro-3-deoxy-D-gluconate-6-phosphate; OAA, oxaloacetate; PEP, phosphoenolpyruvate; PGLU, 6-phospho-Dgluconate; PGLUL, D-glucono- $\delta$-lactone-6-phosphate; R5P, ribose 5-phosphate; RL5P, ribulose 5-phosphate; S7P, sedoheptulose 7-phosphate; 13PG, 1,3-diphosphoglycerate; 3PG, 3-phosphoglycerate; X5P, xylose 5-phosphate. 
Table 1. Strains, plasmids and primers used in this study

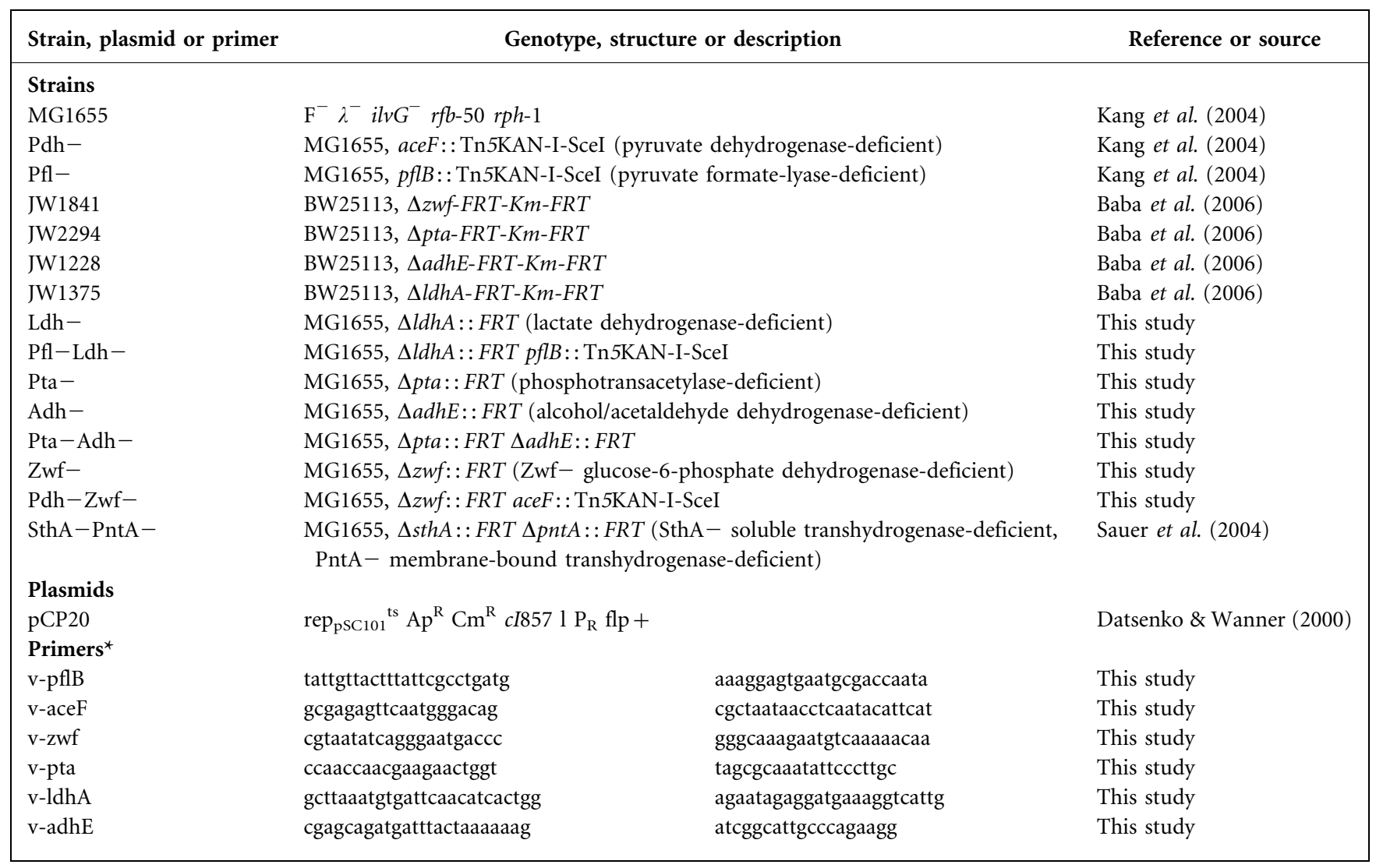

*' $v$ ' indicates primer sequences $\left(5^{\prime}-3^{\prime}\right)$ used for verification purposes. The forward sequence is followed by the reverse sequence in each case. Genes deleted or cloned are apparent from primer names.

MOPS media (pH 7.4) supplemented with 5 g glucuronate $1^{-1}, 10 \mathrm{~g}$ tryptone $1^{-1}$ and $5 \mathrm{~g}$ yeast extract $\mathrm{l}^{-1}$.

Prior to use, strains (stored as glycerol stocks at $-80{ }^{\circ} \mathrm{C}$ ) were streaked onto LB plates (with appropriate antibiotics if required) and incubated overnight at $37{ }^{\circ} \mathrm{C}$ in an Oxoid anaerobic jar with an Oxoid Carbon Dioxide Gas Generating kit.

Analytical methods. $\mathrm{OD}_{550}$ was measured in a BioMate 5 spectrophotometer (Thermo Scientific) and used as an estimate of cell mass $\left(1 \mathrm{OD}_{550}\right.$ unit $=0.34 \mathrm{~g}$ dry weight $\left.\mathrm{l}^{-1}\right)$. After centrifugation, the supernatants were stored at $-20{ }^{\circ} \mathrm{C}$ for HPLC analysis using a Shimadzu Prominence SIL 20 system (Shimadzu Scientific Instruments) equipped with an HPX-87H organic acid column (Bio-Rad). Specific details about the quantification of glucuronate, organic acids, ethanol and hydrogen have been previously reported (Dharmadi et al., 2006; Dharmadi \& Gonzalez, 2005). The identity of fermentation products was verified via $1 \mathrm{D} 1 \mathrm{H}$ NMR, as previously described (Murarka et al., 2008; Dharmadi et al., 2006).

Enzyme activities. Actively growing cells were harvested by centrifugation, washed twice with a saline solution $\left(9 \mathrm{~g} \mathrm{NaCl}^{-1}\right)$ and stored as cell pellets at $-80{ }^{\circ} \mathrm{C}$ until use. Cell pellets were resuspended in $0.1 \mathrm{M}$ potassium phosphate buffer to obtain $\sim 3.4 \mathrm{mg}$ dry cell weight $\mathrm{ml}^{-1}$, and permeabilized with chloroform (Murarka et al., 2008; Tao et al., 2001; Osman et al., 1987). The activity of PDH (toward pyruvate) was monitored spectrophotometrically by following the reduction of $\mathrm{NAD}^{+}$at $340 \mathrm{~nm}$ (Snoep et al., 1990; Danson et al., 1978). The reaction mixture (1 ml, final $\mathrm{pH} 7.0)$ contained
$50 \mathrm{mM}$ potassium phosphate $(\mathrm{pH} 8.0), 2.0 \mathrm{mM}$ sodium pyruvate, $2.5 \mathrm{mM} \mathrm{NAD}{ }^{+}, 1.0 \mathrm{mM} \mathrm{MgCl}, 2.6 \mathrm{mM}$ cystine- $\mathrm{HCl}, 0.13 \mathrm{mM}$ CoA, $0.2 \mathrm{mM}$ thiamine pyrophosphate (Danson et al., 1978) and $50 \mu \mathrm{l}$ permeabilized cells prepared as described above. Linearity of the reactions (protein concentration and time) was established for all preparations. All spectrophotometric measurements were conducted in a BioMate 5 spectrophotometer (Thermo Scientific). The nonenzymic rates were subtracted from the observed initial reaction rates. Enzyme activities are reported as $\mu \mathrm{mol}$ pyruvate oxidized $\mathrm{min}^{-1}$ (mg cell protein $)^{-1}$ and represent averages for at least three cell preparations. Total cell protein was assumed to represent $50 \%$ of the cell dry weight.

NADH/NAD ${ }^{+}$assay. Intracellular levels of NADH and $\mathrm{NAD}^{+}$were measured via the alkaline extraction-reverse phase HPLC method (Stocchi et al., 1985), as previously described (Murarka et al., 2008; Levanon et al., 2005). The fermentation samples were collected in icecold tubes $\left(0{ }^{\circ} \mathrm{C}\right)$ and centrifuged at $3200 \mathrm{~g}$ for $10 \mathrm{~min}$ while maintained under the same conditions. Nucleotides were extracted using the following procedure. Cell pellets were resuspended in $1 \mathrm{ml}$ ice-cold $0.5 \mathrm{M} \mathrm{KOH}$, kept on ice for $10 \mathrm{~min}$ with intermittent vortexing, centrifuged for $10 \mathrm{~min}$ and the supernatant was collected. A $1 \mathrm{ml}$ volume of $1 \mathrm{M} \mathrm{KH}_{2} \mathrm{PO}_{4}$ buffer ( $\mathrm{pH}$ 6.5) was then added to the supernatant and the resulting solution was filtered through a $0.22 \mu \mathrm{m}$ pore-size syringe filter. A $100 \mu \mathrm{l}$ aliquot of this solution was analysed using a Spectra HPLC system (Thermo Separation Products) equipped with a SupelcosilTMLC-18-T column (Supelco). The samples were run with an inorganic mobile phase $(0.1 \mathrm{M}$ 
$\mathrm{KH}_{2} \mathrm{PO}_{4}, \mathrm{pH}$ 6) for 20 min followed by an organic mobile phase (a mixture of $90 \% 0.1 \mathrm{M} \mathrm{KH}_{2} \mathrm{PO}_{4}, \mathrm{pH} 6$, and $10 \%$ methanol). The resulting chromatogram was analysed using the software Chromquest 4.1 (Thermo Electron), thus obtaining the area of the peaks corresponding to concentrations of $\mathrm{NADH}$ and $\mathrm{NAD}^{+}$. The identity of the peaks was verified by analysing samples spiked with known nucleotides.

Calculation of fermentation parameters. Specific growth rates $(\mu$; $\mathrm{h}^{-1}$ ) were estimated by plotting total cell concentration versus time in a log-linear plot. The slope of the curves thus obtained was used as the average specific rate. These curves become straight lines during exponential growth and the calculated specific rate reaches its maximum value $\left(\mu_{\mathrm{m}}\right)$. Specific rates $\left(\mathrm{mmol} \mathrm{g}^{-1}\right.$ cell $\left.^{-1} \mathrm{~h}^{-1}\right)$ during the exponential growth phase were calculated from data for glucuronate consumption and product synthesis and used to demonstrate balanced growth and validity of the steady-state assumption, as previously described (Gonzalez et al., 2003). Growth and product yields (weight or molar basis) were calculated as the amount of cell mass or product synthesized per amount of glucuronate consumed. In the above calculations, an average molecular mass of 25.1 was used, which corresponds to an average E. coli cell of a molecular formula $\mathrm{CH}_{1.81} \mathrm{O}_{0.52} \mathrm{~N}_{0.21}$ (Nielsen et al., 2003).

In silico and in vivo MFA. MFA was conducted using both in silico and in vivo approaches. Flux balance analysis (referred to here as in silico MFA) is a method based on the mass balance constraints in a metabolic network (Edwards \& Palsson, 2000), which can be mathematically represented as:

$S \bullet v=0$

where $S$ is the $m \times n$ stoichiometric matrix, $m$ and $n$ being the number of metabolites and reactions in the network, respectively, and $v$ is a vector containing all the fluxes in the metabolic network. The E. coli model used in this work is based on the pathways known to be active in E. coli during fermentative metabolism in general, as well as those specific to glucuronate utilization (Mandrand-Berthelot et al., 2004; Sawers \& Clark, 2004; Neidhardt et al., 1990, 1996; Keseler et al., 2005; Pramanik \& Keasling, 1997; Stryer, 1995) (see the Reaction Network included in the Supplementary Information available with the online version of this paper). The number of fluxes in the model was 82 and the number of mass balances 74 . A unique distribution of metabolic fluxes was obtained using linear programming (MetaFluxNet) (Lee et al., 2003), whereby the biomass flux was maximized. A list of the reactions included in this analysis along with the details about the construction of the stoichiometric model, the metabolic network and metabolites considered, and the values of estimated fluxes are provided as Supplementary Information with the online version of this paper.

In vivo MFA was conducted using the technique of metabolite balancing as described elsewhere (Stephanopoulos et al., 1998). The metabolic network, and therefore the stoichiometric model, was similar to that described above for in silico MFA, but with 81 reactions, thus resulting in a system with seven degrees of freedom. In this case the available fermentation data were used to calculate seven extracellular fluxes associated with the consumption of glucuronate and the synthesis of fermentation products and biomass. These extracellular fluxes are equivalent to specific rates, and were calculated as described in the section Calculation of fermentation parameters. The availability of extracellular fluxes made the system determined and allowed the calculation of intracellular fluxes without the use of an optimization routine. Three independent measurements were used in the calculation of fluxes, thus allowing the estimation of SDs.

\section{RESULTS}

\section{Cell growth, glucuronate utilization and product synthesis in wild-type MG1655 and strains deficient in pyruvate- and AcCoA-dissimilating enzymes}

The fermentation profiles of MG1655 (wild-type) and $\mathrm{PDH}-(\mathrm{Pdh}-)$ and PFL-deficient $(\mathrm{Pfl}-)$ strains are shown in Fig. 2. No PDH activity was found in mutant Pdh(aceF disrupted), while the lack of fermentative growth on glucose of mutant Pfl- ( $p f l B$ disrupted) demonstrated that PFL was inactive. Both mutants grew much more slowly than the wild-type and reached a lower final cell concentration. Mutant Pdh- also exhibited a well-defined increase in the time required to reach exponential phase. The maximum specific growth rates $\left(\mu_{\mathrm{m}}\right)$ of both mutants were much lower than that of the wild-type: 0.206 and $0.237 \mathrm{~h}^{-1}$ for Pdh - and Pfl-, respectively, compared with $0.354 \mathrm{~h}^{-1}$ for MG1655. The fact that mutant Pfl- is able to grow under fermentative conditions without supplementation of acetate or $\mathrm{CO}_{2}$ is the first such observation, to our knowledge, as E. coli strains devoid of PFL lack the ability to grow fermentatively on other substrates in the absence of the aforementioned supplements (de Graef et al., 1999; Kaiser \& Sawers, 1994; Guest et al., 1989; Varenne et al., 1975).

The profiles of substrate and product concentrations for each strain are also shown in Fig. 2. MG1655 and Pdhhad similar product profiles, although it took twice as much time for the mutant to consume all the substrate and synthesize the products. In both cases a homoacetogeniclike fermentation with co-production of formate was observed, but negligible amounts of succinate and lactate and no ethanol. Significant changes in product profile occurred upon inactivation of PFL (Fig. 2). As expected, there was no accumulation of formate and the generation of acetic acid was significantly lower than in MG1655 and Pdh-. Moreover, there is significant accumulation of lactic acid, reaching levels about 15- and 8-fold higher than those found in the MG1655 and Pdh- cultures, respectively.

No significant changes were observed when the lactate pathway was blocked (deletion of $l d h A$ ) in MG1655, which agrees with earlier reports of no obvious anaerobic growth defects in LDH-deficient mutants (Mat-Jan et al., 1989). However, when $l d h A$ was knocked out in strain Pfl-, no cell growth or glucuronate fermentation was observed. $\mathrm{Pfl}-\mathrm{Ldh}$ - double mutants are known to be unable to grow fermentatively on glucose and other sugars (Mat-Jan et al., 1989).

Acetate synthesis appears to be an important pathway for glucuronate fermentation, as can be inferred by the amounts of acetate produced by all strains (Fig. 2). Acetate is synthesized from AcCoA through the successive action of phosphotransacetylase (PTA; encoded by pta) and acetate kinase (ACK; encoded by ackA), a pathway that generates 

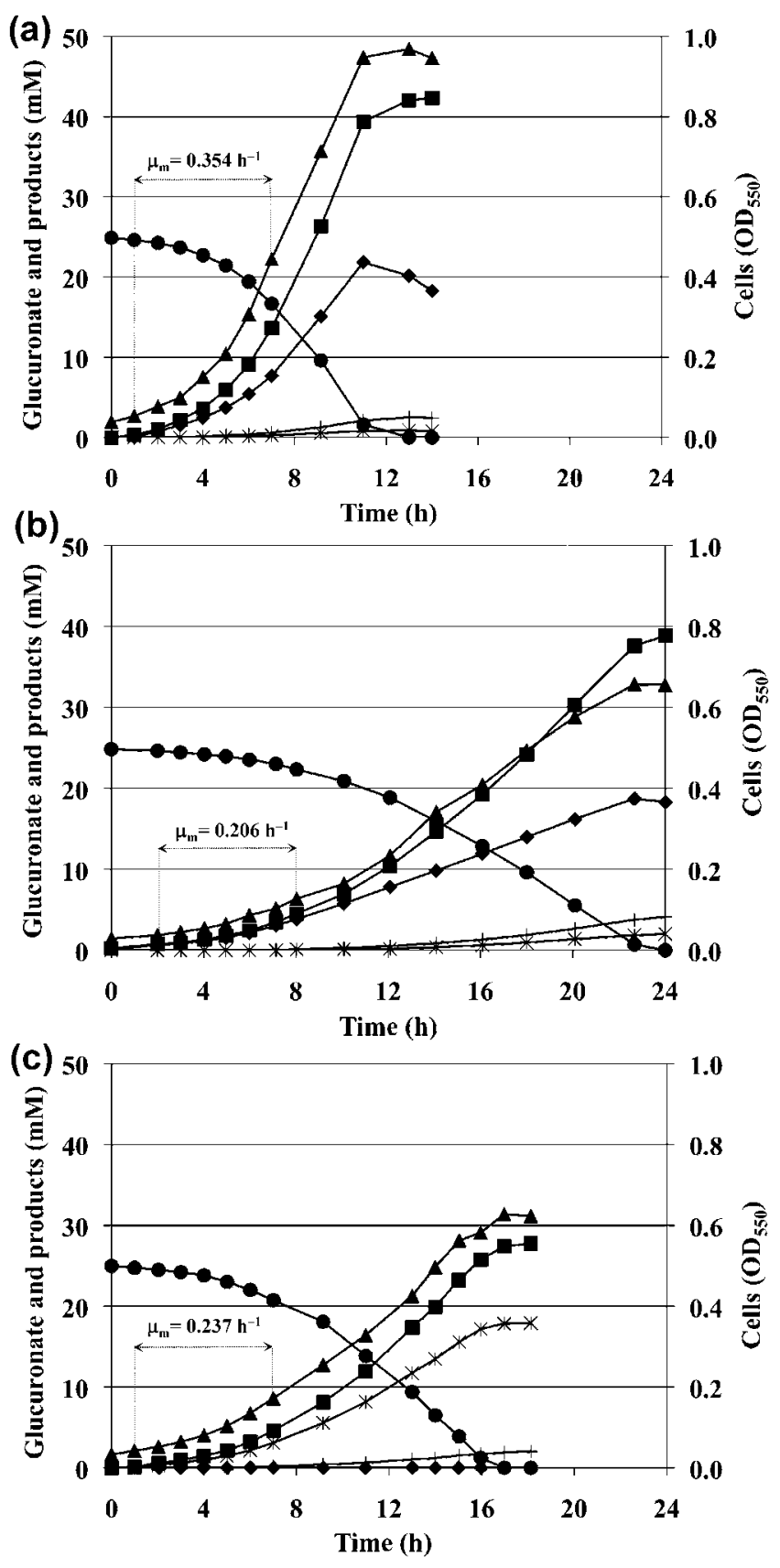

Fig. 2. Cell growth, glucuronate utilization and synthesis of fermentation products in wild-type MG1655 (a) and PDH- and PFL-deficient strains Pdh- (b) and Pfl- (c). Symbols show the concentrations of: $\bullet$, glucuronate; $\boldsymbol{\Delta}$, cells; $\mathbf{\square}$, acetate; *, lactate; + , succinate; formate. The exponential growth phase is indicated by vertical dotted lines and the maximum specific growth rate $\left(\mu_{\mathrm{m}}\right)$ associated with it is also shown.

one molecule of ATP (Fig. 1). PTA-negative mutants do not grow by fermentation of glucose (Guest, 1979) but are able to ferment glycerol (Murarka et al., 2008). In the case of glucuronate, a PTA-deficient mutant $(\mathrm{Pta}-$ strain) did not grow fermentatively on glucuronate and the same results were obtained in a Pta-Ack- double mutant.
While the growth defect of Pta- mutants on glucose is eliminated by a second mutation of adhE (encoding acetaldehyde/alcohol dehydrogenase, Adh) (Gupta \& Clark, 1989), the double mutant Pta-Adh- did not ferment glucuronate. Alcohol dehydrogenase mutants (Adh-) are also impaired in both glucose and glycerol fermentation (Guest, 1979; Gupta \& Clark, 1989; Murarka et al., 2008), but strain Adh - exhibited a phenotype very similar to MG1655 during glucuronate fermentation.

\section{In vivo metabolic flux analysis of wild-type MG1655 and PDH- and PFL-deficient mutants}

Metabolite balancing (Stephanopoulos et al., 1998; Romeo \& Snoep, 2005; Nielsen, 2003), referred to here as in vivo MFA, was used to further investigate the differences between MG1655, Pdh - and Pfl-. The resulting metabolic flux maps for the three strains during exponential growth are shown in Fig. 3.

The flux from pyruvate to AcCoA in MG1655 was split between PFL and PDH. This was a surprising finding, since $\mathrm{PDH}$ is an enzyme primarily involved in respiratory metabolism (Sawers \& Clark, 2004). Indeed, the in vivo activity of $\mathrm{PDH}$ in anaerobic E. coli cultures has been reported as either very low or undetectable (Smith \& Neidhardt 1983; de Graef et al., 1999; Snoep et al., 1993; Lawford \& Rousseau, 1997), and its role in the fermentative metabolism of E. coli has not been established. During fermentation of glucuronate, $\mathrm{PDH}$ could serve as an important source of AcCoA, reducing cofactor $\mathrm{NADH}$ or $\mathrm{CO}_{2}$, all products of the dissimilation of pyruvate via this enzyme. The inactivation of pyruvate-dissimilating enzymes leads to significant redistribution of fluxes throughout the metabolic network, including pathways 'metabolically distant' from the dissimilation of pyruvate (Fig. 3). Since the nature of the metabolic response to the PDH and PFL deficiencies was different, we have categorized the changes as those occurring in either glycolytic and gluconeogenic pathways (i.e. upstream of pyruvate) or fermentative pathways (i.e. downstream of pyruvate) (see Figs 1 and 3).

The fluxes through the glycolytic and gluconeogenic pathways were very similar in strains MG1655 and Pfl-. Most of the glyceraldehyde 3-phosphate generated from the hexuronate dissimilation pathway is channelled to phosphoenolpyruvate/pyruvate, with only a small fraction of the carbon directed through the gluconeogenic and pentose phosphate (PP) pathways (Fig. 3). There are also significant differences between MG1655 and the Pflmutant in the pathways downstream of pyruvate (Fig. 3). The inactivation of PFL is compensated by a 3.5-fold increase in the flux through PDH. The increase in $\mathrm{PDH}$ flux in Pfl- appears to generate large quantities of NADH, whose reoxidation is facilitated by the production of the reduced metabolite lactate (Fig. 3).

The absence of PDH led to significant flux redistribution in the glycolytic and gluconeogenic pathways (Fig. 3). There 

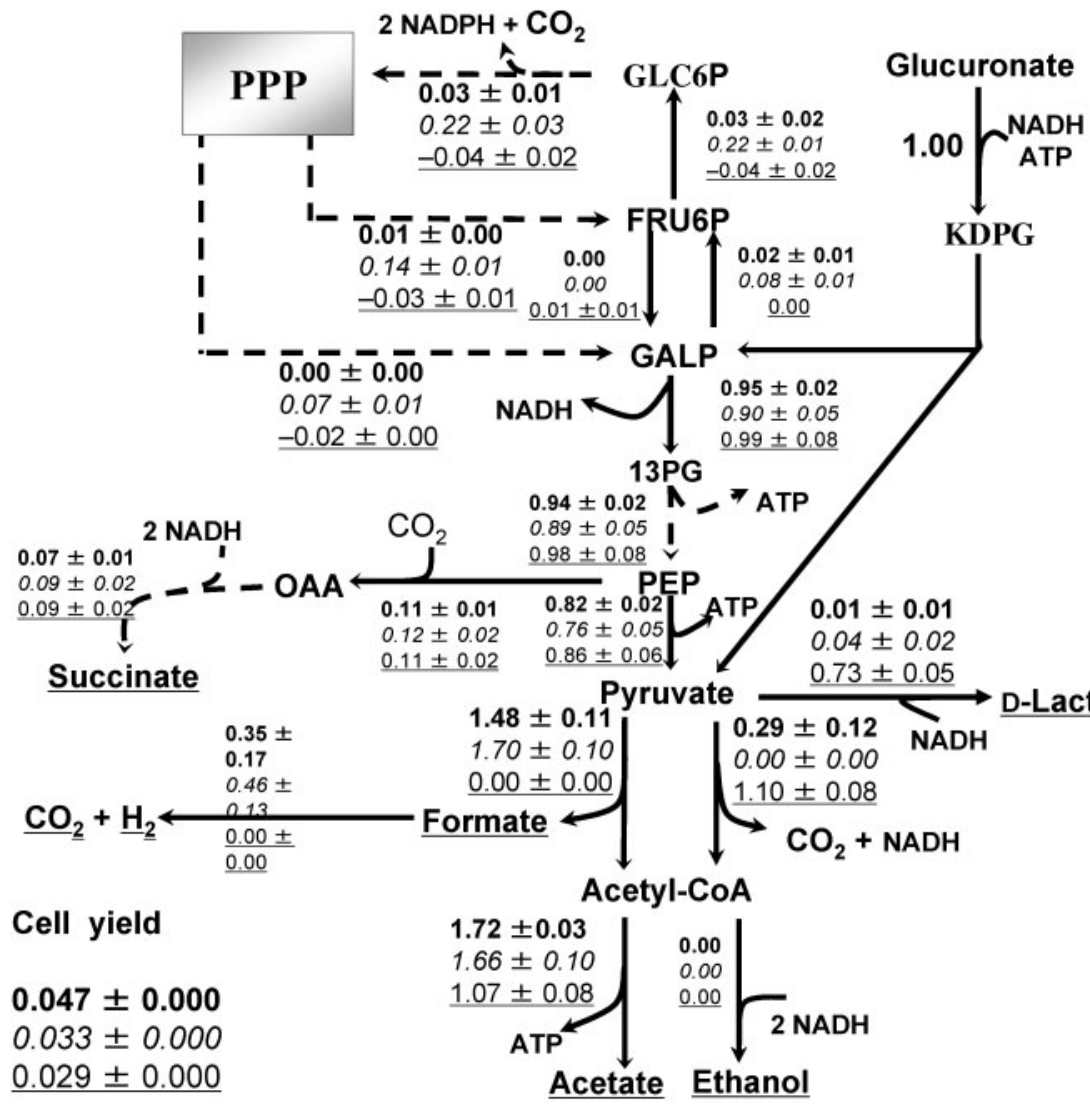

$\mathrm{NADH}+\mathrm{NADP}^{+} \rightarrow \mathrm{NADPH}+\mathrm{NAD}^{+}$

$0.13 \pm 0.07-0.28 \pm 0.05 \underline{0.20 \pm 0.03}$

Fig. 3. In vivo distribution of metabolic fluxes for wild-type MG1655 (in bold type) and mutants Pdh- (in italic type) and Pfl(underlined). Estimated fluxes represent the molar percentages of the average specific glucuronate uptake rates, which were

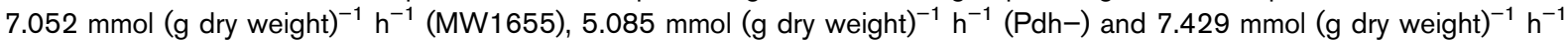
$(\mathrm{Pfl}-)$. The fluxes were calculated for three independent samples taken during the exponential growth phase and means \pm SDS are shown. Fluxes in larger-size font highlight differences between the strains. Arrowheads indicate the direction of fluxes shown as positive (negative fluxes are in the opposite direction to that of arrowheads). Broken lines represent multiple steps. The complete set of fluxes is provided as Supplementary Information. See Fig. 1 and legend for details of the pathways and abbreviations.

was a significant flux originating from glyceraldehyde 3phosphate toward gluconeogenic and PP pathways, which is in contrast with the negligible flux through these pathways in MG1655 and Pfl-. However, a major fraction of the oxidative pentose phosphate pathway (ox-PPP) flux in mutant $\mathrm{Pdh}-$ cycled back to glyceraldehyde 3phosphate and fructose 6-phosphate via the non-oxidative pentose phosphate pathway (non-ox-PPP). The significant increase in flux through the ox-PPP in mutant $\mathrm{Pdh}-$ is worth investigating, since the ox-PPP, like PDH (which is inactivated in strain $\mathrm{Pdh}-$ ), generates $\mathrm{CO}_{2}$ and reducing equivalents.

The transhydrogenase flux shown in Fig. 3 corresponds to the interconversion of NADPH and NADH catalysed by the soluble and membrane-bound transhydrogenases SthA and PntAB, respectively (Sauer et al., 2004; Canonaco et al., 2001). This flux is positive in MG1655 and in Pfl-, implying that the reduced cofactor $\mathrm{NADH}$ is converted to the biosynthetic reducing power NADPH. The NADH required for this reaction is met by the flux through $\mathrm{PDH}$. Accordingly, higher flux through $\mathrm{PDH}$ in strain Pfl- led to a $54 \%$ increase in the transhydrogenase flux (Fig. 3). This interconversion, however, is working in the opposite direction in strain $\mathrm{Pdh}-$, where the $\mathrm{PDH}$ pathway is inactive and excess NADPH generated via the ox-PPP requires conversion to $\mathrm{NADH}$.

\section{In silico MFA suggests the basis for the differences in flux distribution among strains MG1655, Pdh- and PfI-}

Metabolic fluxes calculated via in vivo MFA portrayed major flux redistributions in response to the PFL and PDH deficiencies (Fig. 3). To further analyse this redistribution 
of fluxes we used flux balance analysis (Edwards \& Palsson, 2000), a technique widely applied to the study of microbial metabolism (Downs, 2006; Romeo \& Snoep, 2005; Nielsen, 2003), and referred to here as in silico MFA. To this end, simulations were conducted in different scenarios by selectively relaxing or enforcing redox (R) and ATP (A) constraints (see Table 2 and Supplementary Information for details).

Key features of the distribution of metabolic fluxes obtained via in silico MFA are summarized in Table 2. The $A_{C} R_{C}$ 'simulated scenario' indicates that the system is subjected to both ATP and redox constraints (all scenarios are subjected to carbon constraints). Accordingly, simulations conducted under $A_{C} R_{C}$ scenarios should resemble the flux distributions calculated via in vivo MFA (Fig. 3). A comparison of the in vivo fluxes (Fig. 3) with the in silico fluxes shown in Table $2\left(\mathrm{~A}_{\mathrm{C}} \mathrm{R}_{\mathrm{C}}\right.$ simulated scenarios) validates the ability of the in silico approach to reveal key characteristics of glucuronate fermentation in wild-type and PDH- and PFL-deficient strains. Further simulations were conducted in scenarios in which redox (R) and/or ATP (A) constraints were relaxed and the results are discussed below.

For the wild-type network, most fluxes remained unchanged, including the cell yield, if the redox balance constraint was relieved $\left(\mathrm{R}_{\mathrm{U}}\right.$ scenario, unconstrained redox $)$ (Table 2). In contrast, the cell yield increased more than fivefold upon relaxing the constraint on ATP availability $\left(A_{U}\right.$ scenario, unconstrained ATP), which then increased further upon relaxing the redox constraint $\left(A_{U} R_{U}\right.$ scenario, unconstrained ATP and redox). In all simulated scenarios there was a large flux through PDH, while the ox-PPP flux was observed only in the $A_{U}$ case (Table 2). This analysis suggests that the growth of wild-type cells is limited by ATP availability, and that the requirements for reducing equivalents are fulfilled by adjusting metabolism to use either PDH or the ox-PPP. Our experimental results suggested that the ATP required for cell growth is provided by the synthesis of acetate through the PTA-ACK pathway; i.e. a Pta - strain, which is unable to synthesize acetate, did not ferment glucuronate. The in silico analysis of a Ptadeficient mutant further supported this hypothesis, as this strain did not grow or ferment glucuronate but recovered the ability to grow on an unconstrained ATP scenario (see Supplementary Information).

As discussed in the sections above, the inactivation of $\mathrm{PDH}$ led to an increase in the ox-PPP flux, a pathway that like $\mathrm{PDH}$ generates reducing equivalents and $\mathrm{CO}_{2}$ (but no ATP is involved in these pathways). In silico and in vivo MFA (Table 2, $A_{C} R_{C}$ scenario, Fig. 3) show that this additional flux through the ox-PPP in strain Pdh- is cycled back to the glycolytic intermediates glyceraldehyde 3-phosphate and fructose 6-phosphate. Consequently, it can be inferred that the high ox-PPP flux does not benefit the cells by providing PPP intermediates. One possible explanation for the increase in the ox-PPP flux is that it fulfils metabolic requirements for either $\mathrm{CO}_{2}$ or $\mathrm{NAD}(\mathrm{P}) \mathrm{H}$. However, since our simulations show that there is a net generation of $\mathrm{CO}_{2}$ in all strains and conditions (shown in Supplementary Information), it can be hypothesized that the cells increase the ox-PPP flux to generate reducing equivalents. Even with the increase in the ox-PPP flux, the cell yield in Pdhis lower than in the wild-type. However, relaxing the constraint of redox balance in mutant Pdh- leads to equalization of fluxes with those in the wild-type (Table 2, $\mathrm{R}_{\mathrm{U}}$ scenario). Also, relaxing the ATP constraint in Pdhleads to a lower cell yield than a similar situation in the wild-type ( $A_{U}$ scenarios for MG1655 and Pdh-).

The analysis of mutant Pfl- in an unconstrained redox scenario $\left(R_{U}\right)$ predicts a biomass yield equal to the wildtype, exclusive dissimilation of pyruvate via $\mathrm{PDH}$, no lactate co-production, and a higher acetate flux (Table 2). These results suggest that the presence of PFL in wild-type E. coli enables the cells to maintain high flux through an ATP-generating pathway (phosphotransacetylase-ACK), while generating the required $\mathrm{NADH}$ via $\mathrm{PDH}$. In the absence of PFL, pyruvate is dissimilated to AcCoA via $\mathrm{PDH}$, generating extra reducing equivalents and thus requiring the synthesis of lactate to maintain redox poise. However, lactate is a suboptimal route in terms of ATP production, hence the lower cell yield.

The transhydrogenase fluxes for the ' $A_{C} R_{C}$ ' and ' $A_{U}$ ' scenarios in strain $\mathrm{Pdh}-$ are negative (Table 2), indicating that the NADPH generated via the ox-PPP is more than that required for biosynthetic functions and that the excess is converted to NADH. The cell yield for these simulations is lower than the wild-type under the same simulated scenario, implying that the ox-PPP is unable to completely compensate for the loss of PDH. Since the ox-PPP, like $\mathrm{PDH}$, generates reducing equivalents, we simulated the effect of inactivating this pathway by deleting the gene $z w f$, which encodes the enzyme that catalyses the first step of the ox-PPP (glucose-6-phosphate dehydrogenase). The performance of mutant $\mathrm{Zwf}-$ is predicted to be indistinguishable from that of MG1655, exhibiting similar growth yield and distribution of metabolic fluxes (Table $2, \mathrm{~A}_{\mathrm{C}} \mathrm{R}_{\mathrm{C}}$ scenario). However, the ox-PPP becomes essential for cell growth upon deletion of PDH (Table 2, double mutant $\mathrm{Pdh}-\mathrm{Zwf}-, \mathrm{A}_{\mathrm{C}} \mathrm{R}_{\mathrm{C}}$ scenario). Additionally, this double mutant is predicted to regain wild-type growth characteristics when redox constraints are relaxed (Table 2, $R_{U}$ scenario). In contrast, relaxing the constraints on ATP availability does not lead to a recovery of $\mathrm{Pdh}-\mathrm{Zwf}-$ (Table 2, $\mathrm{A}_{\mathrm{U}}$ scenario).

\section{Experimental evidence supporting the role of PDH in the fermentative metabolism of glucuronate}

The in silico and in vivo analyses conducted in previous sections led to the hypothesis that the role of PDH in the fermentative metabolism of glucuronate is to generate reducing equivalents. Although $\mathrm{PDH}$ generates both $\mathrm{CO}_{2}$ and reducing equivalents, the in silico analysis predicted 
Table 2. In silico MFA of wild-type $E$. coli and mutants deficient in PDH (Pdh-), PFL (Pfl-) and glucose-6-phosphate dehydrogenase (Zwf-) enzymes

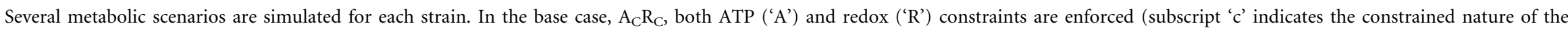

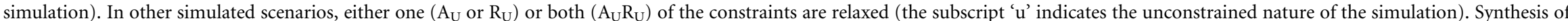

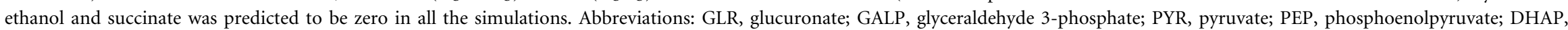
dihydroxyacetone phosphate; FRU6P, fructose 6-phosphate; GLC6P; glucose 6-phosphate; RL5P, ribulose 5-phosphate; R5P, ribose 5-phosphate; X5P, xylose 5-phosphate; E4P, erythrose 4phosphate. See Fig. 1 for more details about pathways. Fluxes were normalized with respect to the glycerol consumption flux (on a molar basis).

\begin{tabular}{|c|c|c|c|c|c|c|c|c|c|c|c|c|c|}
\hline \multirow[t]{3}{*}{ Pathway or reaction } & \multicolumn{13}{|c|}{ Strain and simulated scenario } \\
\hline & \multicolumn{4}{|c|}{ Wild-type MG1655 } & \multicolumn{3}{|c|}{ Pdh- } & \multicolumn{2}{|c|}{ Pfl- } & \multirow{2}{*}{$\begin{array}{l}\text { Zwf- } \\
A_{C} R_{C}\end{array}$} & \multicolumn{3}{|c|}{ Pdh-Zwf- } \\
\hline & $\mathbf{A}_{\mathbf{C}} \mathbf{R}_{\mathbf{C}}$ & $\mathbf{R}_{\mathbf{U}}$ & $\mathbf{A}_{\mathbf{U}}$ & $\mathbf{A}_{\mathbf{U}} \mathbf{R}_{\mathbf{U}}$ & $\mathbf{A}_{\mathbf{C}} \mathbf{R}_{\mathbf{C}}$ & $\mathbf{R}_{\mathbf{U}}$ & $\mathbf{A}_{\mathbf{U}}$ & $\mathbf{A}_{\mathrm{C}} \mathbf{R}_{\mathrm{C}}$ & $\mathbf{R}_{\mathbf{U}}$ & & $\mathbf{A}_{\mathrm{C}} \mathbf{R}_{\mathrm{C}}$ & $\mathbf{R}_{\mathrm{U}}$ & $A_{U}$ \\
\hline \multicolumn{14}{|l|}{ Glucuronate utilization } \\
\hline $\begin{array}{l}\mathrm{GLR}+\mathrm{ATP}+\mathrm{NADH} \rightarrow \mathrm{GALP}+\mathrm{PYR} \\
\text { Embden-Meyerhof-Parnas }\end{array}$ & 1.000 & 1.000 & 1.000 & 1.000 & 1.000 & 1.000 & 1.000 & 1.000 & 1.000 & 1.000 & 1.000 & 1.000 & 1.000 \\
\hline $\mathrm{GALP} \longrightarrow \longrightarrow \mathrm{PEP}+\mathrm{NADH}+\mathrm{ATP}$ & 0.947 & 0.947 & 0.536 & 0.653 & 0.894 & 0.947 & 0.471 & 0.961 & 0.947 & 0.947 & 1.000 & 0.947 & 1.000 \\
\hline $\mathrm{PEP} \rightarrow \mathrm{PYR}+\mathrm{ATP}$ & 0.847 & 0.847 & 0.000 & 0.000 & 0.800 & 0.847 & 0.000 & 0.889 & 0.847 & 0.847 & 1.000 & 0.847 & 1.000 \\
\hline \multicolumn{14}{|l|}{ Gluconeogenesis } \\
\hline DHAP + GALP $\rightarrow$ FRU6P & 0.020 & 0.020 & 0.285 & 0.130 & 0.074 & 0.020 & 0.372 & 0.014 & 0.020 & 0.020 & 0.000 & 0.020 & 0.000 \\
\hline $\begin{array}{l}\text { FRU6P } \rightarrow \text { GLC6P } \\
\text { ox-PPP }\end{array}$ & 0.004 & 0.004 & 0.555 & 0.026 & 0.171 & 0.004 & 0.000 & 0.003 & 0.004 & 0.004 & 0.000 & 0.004 & 3.000 \\
\hline $\begin{array}{l}\mathrm{GLC} 6 \mathrm{P} \rightarrow \mathrm{RL} 5 \mathrm{P}+\mathrm{CO}_{2}+\mathrm{NADPH} \\
\text { Non-ox-PPP }\end{array}$ & 0.000 & 0.000 & 0.534 & 0.000 & 0.167 & 0.000 & 0.837 & 0.000 & 0.000 & - & - & - & - \\
\hline $\mathrm{R} 5 \mathrm{P}+\mathrm{X} 5 \mathrm{P} \rightarrow \mathrm{E} 4 \mathrm{P}+\mathrm{FRU6P}$ & -0.004 & -0.004 & 0.158 & -0.024 & 0.052 & -0.004 & 0.262 & -0.003 & -0.004 & -0.004 & 0.000 & -0.004 & 0.000 \\
\hline $\mathrm{X} 5 \mathrm{P}+\mathrm{E} 4 \mathrm{P} \rightarrow \mathrm{GALP}+\mathrm{FRU6P}$ & -0.011 & -0.011 & 0.120 & -0.071 & 0.046 & -0.011 & 0.228 & -0.008 & -0.011 & -0.011 & 0.000 & -0.011 & 0.000 \\
\hline \multicolumn{14}{|l|}{ Pyruvate dissimilation } \\
\hline $\mathrm{PYR} \rightarrow \mathrm{AcCoA}+\mathrm{FORMATE}$ & 1.496 & 0.000 & 0.000 & 0.000 & 1.747 & 1.791 & 0.736 & - & - & 1.496 & 2.000 & 1.791 & 2.000 \\
\hline $\begin{array}{l}\mathrm{PYR} \rightarrow \mathrm{AcCoA}+\mathrm{CO}_{2}+\mathrm{NADH} \\
\text { Fermentation }\end{array}$ & 0.295 & 1.791 & 0.699 & 0.633 & - & - & - & 1.032 & 1.791 & 0.295 & - & - & - \\
\hline $\mathrm{PYR}+\mathrm{NADH} \rightarrow \mathrm{LAC}$ & 0.000 & 0.000 & 0.000 & 0.000 & 0.000 & 0.000 & 0.000 & 0.817 & 0.000 & 0.000 & 0.000 & 0.000 & 0.000 \\
\hline $\mathrm{AcCoA} \rightarrow \mathrm{AC}+\mathrm{ATP}$ & 1.712 & 1.712 & 0.270 & 0.112 & 1.672 & 1.712 & 0.359 & 0.974 & 1.712 & 1.712 & 2.000 & 1.712 & 2.000 \\
\hline \multicolumn{14}{|l|}{ Transhydrogenases } \\
\hline $\begin{array}{l}\mathrm{NADH}+\mathrm{NADP}^{+} \rightarrow \mathrm{NAD}^{+}+\mathrm{NADPH} \\
\text { Cell growth }\end{array}$ & 0.313 & 0.313 & 0.618 & 2.053 & -0.039 & 0.313 & -0.193 & 0.228 & 0.313 & 0.313 & 0.000 & 0.313 & 0.000 \\
\hline Precursor metabolites $\rightarrow$ Biomass & 0.135 & 0.135 & 0.726 & 0.884 & 0.128 & 0.135 & 0.637 & 0.098 & 0.135 & 0.135 & 0.000 & 0.135 & 0.000 \\
\hline
\end{tabular}


that the cultures are not limited by $\mathrm{CO}_{2}$, hence the hypothesized role. Experiments in which sodium bicarbonate was included in the medium resulted in fermentation profiles essentially indistinguishable from those presented in Fig. 2 (data not shown).

Given the proposed role of PDH in the generation of reducing equivalents, we assessed the internal redox state of MG1655, $\mathrm{Pdh}-$ and $\mathrm{Pfl}-$ by measuring the $\mathrm{NADH}: \mathrm{NAD}^{+}$ratio (Table 3 ). As can be inferred from the results, the internal redox state of the cells is significantly affected by the choice of pyruvate-dissimilating enzyme. The disruption of $\mathrm{PDH}$ was associated with a $30 \%$ decrease in the $\mathrm{NADH}: \mathrm{NAD}^{+}$ratio, when compared with that measured in MG1655. The knockout of PFL, on the other hand, was accompanied by a more than $40 \%$ increase in this ratio. Overall, the NADH: $\mathrm{NAD}^{+}$ratio was twofold higher in mutant $\mathrm{Pfl}-$ when compared with $\mathrm{Pdh}-$. The changes in NADH: $\mathrm{NAD}^{+}$ratio (Table 3) correlated well with the observed changes in PDH fluxes (Fig. 3).

In the absence of PDH (i.e. mutant $\mathrm{Pdh}-$ ), the flux analysis indicates that the ox-PPP produces the reducing equivalents otherwise generated by $\mathrm{PDH}$, although the slower growth of Pdh- appears to indicate that the ox-PPP is less efficient in fulfilling this function. This led to the prediction that a deficiency in the ox-PPP would have no effect on cell growth in the wild-type background but would be detrimental when combined with a PDH deficiency (see Table 2, single and double mutants Zwfand Pdh-Zwf-). Strains Zwf- and Pdh-Zwf- were then constructed and their fermentative growth on glucuronate was evaluated. While strain $\mathrm{Zwf}-$ grew at a maximum specific growth rate equivalent to that observed for MG1655, a $32 \%$ reduction in this parameter was observed when $z w f$ was disrupted in the PDH-deficient strain (Table 3). This double mutant grew very slowly (Table 3), exhibiting a lag time in fermenters of about $5 \mathrm{~h}$ and taking $28 \mathrm{~h}$ to reach stationary phase (data not shown). Neither the activity of PDH nor the internal redox state of the cells (NADH: $\mathrm{NAD}^{+}$ratio) was significantly affected by the inactivation of Zwf in MG1655 (Table 3). However, a significant decrease in $\mathrm{NADH}: \mathrm{NAD}^{+}$ratio was observed upon Zwf inactivation in strain Pdh-. Note that PDH inactivation in MG1655 also led to a low
$\mathrm{NADH}: \mathrm{NAD}^{+}$, although the decrease was less pronounced than in double mutant Pdh-Zwf- (Table 3). As in the case of mutant Pdh-, supplementation of the medium with bicarbonate did not improve the performance of strain $\mathrm{Pdh}-\mathrm{Zwf}-$, which suggests no $\mathrm{CO}_{2}$ limitation (data not shown). These results indicate that both PDH and the ox-PPP are major sources of reducing equivalents, although the ox-PPP appears to make a significant contribution only in the absence of PDH.

If the reducing equivalents generated by $\mathrm{PDH}$ (i.e. NADH) were to play an important role as a source of biosynthetically useful reducing power (i.e. NADPH), they would need to be converted to NADPH by the action of transhydrogenases. In agreement with this reasoning, transhydrogenase-deficient strain SthA-PntA- (SthA-, soluble transhydrogenase-deficient; PntA-, membrane-bound transhydrogenase-deficient) exhibited a $33 \%$ decrease in $\mu_{\mathrm{m}}\left(0.229 \mathrm{~h}^{-1}\right)$ when compared with MG1655. While this decrease in $\mu_{\mathrm{m}}$ was significant, it was not as large a decrease as that observed for $\mathrm{Pdh}-(45 \%)$. Moreover, strain SthA-PntA - did not exhibit a significant delay in its transition into the exponential phase, although $\mathrm{PDH}-$ deficient strains did.

\section{DISCUSSION}

In the work reported here, in silico and in vivo MFA were used to investigate the fermentative metabolism of glucuronate in E. coli, with emphasis on pyruvatedissimilation pathways. This analysis revealed that both PFL and PDH mediate the dissimilation of pyruvate. This is in contrast with previous reports of low (or undetectable) in vivo activity of PDH in anaerobic $E$. coli cultures grown on other carbon sources (Sawers \& Clark, 2004; Smith \& Neidhardt, 1983; de Graef et al., 1999; Snoep et al., 1993; Lawford \& Rousseau, 1997). An even more surprising result was the finding that PDH can support fermentative growth on glucuronate in the absence of PFL. This was unexpected because PFL-deficient strains are unable to ferment glucose and other substrates in the absence of $\mathrm{CO}_{2}$ or acetate (Hasona et al., 2004; Sawers \& Clark, 2004; de Graef et al., 1999; Varenne et al., 1975). Another interesting finding was that the homoacetogenic nature of glucuronate

Table 3. Maximum specific growth rate $\left(\mu_{\mathrm{m}}\right)$, internal redox state (NADH : NAD ${ }^{+}$ratio) and activity of PDH in wild-type MG1655 and mutants deficient in PDH (Pdh-), PFL (Pfl-) and glucose-6-phosphate dehydrogenase (Zwf-) enzymes

Cells were grown in Hungate tubes using MOPS minimal medium with $5 \mathrm{~g}$ glucuronate $\mathrm{1}^{-1}$, as described in Methods.

\begin{tabular}{|c|c|c|c|}
\hline Strain & $\mu_{\mathrm{m}}\left(\mathrm{h}^{-1}\right)$ & $\mathrm{NADH}: \mathrm{NAD}^{+}$(ratio) & PDH activity $\left[\mu \mathrm{mol}(\mathrm{mg} \text { cell protein })^{-1} \mathrm{~min}^{-1}\right]$ \\
\hline $\mathrm{Pdh}-$ & $0.206 \pm 0.005$ & $0.089 \pm 0.011$ & 0.00 \\
\hline Zwf- & $0.331 \pm 0.007$ & $0.136 \pm 0.019$ & $0.34 \pm 0.03$ \\
\hline $\mathrm{Pdh}-\mathrm{Zwf}-$ & $0.130 \pm 0.003$ & $0.063 \pm 0.004$ & 0.00 \\
\hline
\end{tabular}


fermentation in the wild-type strain shifted to a mixed lactic and acetic acid fermentation upon deletion of the PFL pathway. While both Pdh - and Pfl- exhibited lower specific growth rates than MG1655, a slower transition into the exponential phase was only observed in the case of Pdh-. Finally, a significant redistribution of metabolic fluxes was found upon perturbation of pyruvate metabolism by PFL and PDH disruptions. Since no physiological role had been proposed for $\mathrm{PDH}$ in the fermentative metabolism of E. coli, we further investigated the metabolic differences between strains MG1655 and Pdh-.

In silico and in vivo MFA were used to help establish the role of PDH. In vivo MFA revealed an increase in the oxPPP flux in mutant Pdh-, which was postulated to be the compensatory mechanism that the cells use to generate reducing equivalents in the absence of $\mathrm{PDH}$; i.e. $\mathrm{PDH}$ and the ox-PPP are the two primary sources of reducing equivalents. Consequently, the in silico MFA predicted that blocking the ox-PPP would make the negative effect of the $\mathrm{PDH}$ deficiency more pronounced (Table 2). This analysis led to the hypothesis that the role of PDH was to supply reducing equivalents. The decrease in $\mathrm{NADH}: \mathrm{NAD}^{+}$ratio in the PDH-deficient strain (Table 3) indicates that PDH indeed plays an important role in maintaining the internal redox state. Moreover, a $z w f$ deletion (which blocked the ox-PPP) had no growth phenotype when introduced in MG1655 but made the characteristic phenotype of a PDH deficiency more pronounced when introduced into strain $\mathrm{Pdh}-$ (i.e. slower transition into the exponential phase and lower specific growth rate). A Pdh-Zwf- double mutant exhibited a NADH: $\mathrm{NAD}^{+}$ratio even lower than that of Pdh- (Table 3).

The importance of the reducing equivalents generated by $\mathrm{PDH}$ is twofold. First, the dissimilation of pyruvate through PDH leads to an internal redox state favourable to the conversion of glucuronate to the glycolytic intermediates glyceraldehyde 3-phosphate and pyruvate, a pathway that consumes reducing equivalents (Fig. 1). The metabolic link between the PDH-mediated dissimilation of pyruvate and glucuronate utilization can be revealed through the analysis of the proposed role of $\mathrm{PDH}$ and the kinetic properties of D-mannonate reductase, a key enzyme in glucuronate utilization (Fig. 1) (MandrandBerthelot et al., 2004). D-mannonate reductase exhibits typical Michaelis-Menten kinetics with respect to NADH $\left(K_{\mathrm{m}}=0.03 \mathrm{mM}\right)$ and is inhibited by $\mathrm{NAD}^{+}$(MandrandBerthelot \& Lagarde, 1977). By generating the appropriate internal redox state (i.e. sufficiently high NADH and/or low $\mathrm{NAD}^{+}$), $\mathrm{PDH}$ ensures the functioning of D-mannonate oxidoreductase and therefore the utilization of glucuronate. On the other hand, given the redox-neutral nature of the overall pathway that converts glucuronate into pyruvate (Fig. 1), the generation of reducing equivalents by $\mathrm{PDH}$ could enable biosynthetic functions such as the synthesis of building blocks, macromolecules and ultimately cell mass. In this role, PDH works in partnership with transhydrogenases, which convert the
NADH generated by PDH into biosynthetically useful NADPH (Sauer et al., 2004; Fuhrer \& Sauer, 2009).

While the role of $\mathrm{PDH}$ as a source of reducing equivalents is supported by the results presented here, one might question why $\mathrm{PDH}$ is preferred for this purpose over the ox-PPP. The equations below compare the overall stoichiometry of each pathway, assuming equal $\mathrm{NAD}(\mathrm{P}) \mathrm{H}$ yields and the interconversion of $\mathrm{NADH}$ and $\mathrm{NADPH}$ via transhydrogenases:

PDH:

6glucuronate $\rightarrow$

$12 \mathrm{AcCoA}+5 \mathrm{CO}_{2}+7$ formate $+5 \mathrm{NAD}(\mathrm{P}) \mathrm{H}+6 \mathrm{ATP}$

ox-PPP:

6glucuronate $\rightarrow$

$11 \mathrm{AcCoA}+3 \mathrm{CO}_{2}+11$ formate $+5 \mathrm{NAD}(\mathrm{P}) \mathrm{H}+4 \mathrm{ATP}$

As can be seen, for the same amount of reducing equivalents generated upon consuming an equal amount of glucuronate, PDH generates more ATP (energy) and more AcCoA (carbon). Cells utilize both energy and carbon to generate the building blocks of biomass, and our in silico analysis suggests that glucuronate fermentation is limited by ATP availability. Therefore, PDH is more energy- and carbon-efficient than the ox-PPP, and this could be the reason why wild-type MG1655 prefers PDH to fulfil its needs for reducing equivalents and why $\mathrm{PDH}$ deficient strains grow more slowly even when the ox-PPP is available to substitute for this function. An important role for PDH in the fermentative metabolism of Enterococcus faecalis during the utilization of a similarly oxidized substrate, pyruvate, has been reported (Snoep et al., 1990).

\section{ACKNOWLEDGEMENTS}

We thank the University of Wisconsin E. coli Genome Project (Madison, WI), the National BioResource Project (National Institute of Genetics, Japan), and K.-Y. San, Rice University, and U. Sauer for providing research materials. This work was partially supported by a grant from the US National Science Foundation (BES-0331388/BES0601549).

\section{REFERENCES}

Atsumi, S. \& Liao, J. C. (2008). Metabolic engineering for advanced biofuels production from Escherichia coli. Curr Opin Biotechnol 19, 414-419.

Baba, T., Ara, T., Hasegawa, M., Takai, Y., Okumura, Y., Baba, M., Datsenko, K. A., Tomita, M., Wanner, B. L. \& Mori, H. (2006). Construction of Escherichia coli K-12 in-frame, single-gene knockout mutants: the Keio collection. Mol Syst Biol 2, :2006.0008

Canonaco, F., Hess, T. A., Heri, S., Wang, T., Szyperski, T. \& Sauer, U. (2001). Metabolic flux response to phosphoglucose isomerase knockout in Escherichia coli and impact of overexpression of the soluble transhydrogenase UdhA. FEMS Microbiol Lett 204, 247-252.

Danson, M. J., Hooper, E. A. \& Perham, R. N. (1978). Intramolecular coupling of active sites in the pyruvate dehydrogenase multienzyme complex of Escherichia coli. Biochem J 175, 193-198. 
Datsenko, K. A. \& Wanner, B. L. (2000). One-step inactivation of chromosomal genes in Escherichia coli K-12 using PCR products. Proc Natl Acad Sci U S A 97, 6640-6645.

de Graef, M. R., Alexeeva, S., Snoep, J. L. \& Teixeira de Mattos, M. J. (1999). The steady-state internal redox state (NADH/NAD) reflects the external redox state and is correlated with catabolic adaptation in Escherichia coli. J Bacteriol 181, 2351-2357.

Dharmadi, Y. \& Gonzalez, R. (2005). A better global resolution function and a novel iterative stochastic search method for optimization of high-performance liquid chromatographic separation. J Chromatogr A 1070, 89-101.

Dharmadi, Y., Murarka, A. \& Gonzalez, R. (2006). Anaerobic fermentation of glycerol by Escherichia coli: a new platform for metabolic engineering. Biotechnol Bioeng 94, 821-829.

Downs, D. M. (2006). Understanding microbial metabolism. Annu Rev Microbiol 60, 533-559.

Edwards, J. S. \& Palsson, B. O. (2000). The Escherichia coli MG1655 in silico metabolic genotype: its definition, characteristics, and capabilities. Proc Natl Acad Sci U S A 97, 5528-5533.

Finegold, S. M., Sutter, V. L. \& Mathisen, G. E. (1983). Normal indigenous intestinal flora. In Human Intestinal Microflora in Health and Disease, pp. 3-31. Edited by D. J. Hentges. New York: Academic Press.

Fuhrer, T. \& Sauer, U. (2009). Different biochemical mechanisms ensure network-wide balancing of reducing equivalents in microbial metabolism. J Bacteriol 191, 2112-2121.

Gonzalez, R., Andrews, B. A., Molitor, J. \& Asenjo, J. A. (2003). Metabolic analysis of the synthesis of high levels of intracellular human SOD in Saccharomyces cerevisiae rhSOD 2060411 SGA122. Biotechnol Bioeng 82, 152-169.

Guest, J. R. (1979). Anaerobic growth of Escherichia coli K-12 with fumarate as terminal electron acceptor. Genetic studies with menaquinone and fluoracetate resistant mutants. J Gen Microbiol 115, 259-271.

Guest, J. R., Angier, S. J. \& Russell, G. C. (1989). Structure, expression, and protein engineering of the pyruvate dehydrogenase complex of Escherichia coli. Ann N Y Acad Sci 573, 76-99.

Gupta, S. \& Clark, D. P. (1989). Escherichia coli derivatives lacking both alcohol dehydrogenase and phosphotransacetylase grow anaerobically by lactate fermentation. J Bacteriol 171, 3650-3655.

Hasona, A., Kim, Y., Healy, F. G., Ingram, L. O. \& Shanmugam, K. T. (2004). Pyruvate formate lyase and acetate kinase are essential for anaerobic growth of Escherichia coli on xylose. J Bacteriol 186, 75937600.

Kaiser, M. \& Sawers, G. (1994). Pyruvate formate-lyase is not essential for nitrate respiration by Escherichia coli. FEMS Microbiol Lett 117, 163-168.

Kang, Y. S., Durfee, T., Glasner, J. D., Qiu, Y., Frisch, D., Winterberg, K. M. \& Blattner, F. R. (2004). Systematic mutagenesis of the Escherichia coli genome. J Bacteriol 186, 4921-4930.

Keseler, I. M., Collado-Vides, J., Gama-Castro, S., Ingraham, J., Paley, S., Paulsen, I. T., Peralta-Gil, M. \& Karp, P. D. (2005). EcoCyc: a comprehensive database resource for Escherichia coli. Nucleic Acids Res 33, D334-D337.

Lawford, H. G. \& Rousseau, J. D. (1997). Fermentation of biomassderived glucuronic acid by pet expressing recombinants of Escherichia coli B. Appl Biochem Biotechnol 63-65, 221-241.

Lee, D. Y., Yun, H., Park, S. \& Lee, S. Y. (2003). MetaFluxNet: the management of metabolic reaction information and quantitative metabolic flux analysis. Bioinformatics 19, 2144-2146.
Levanon, S. S., San, K. Y. \& Bennett, G. N. (2005). Effect of oxygen on the Escherichia coli ArcA and FNR regulation systems and metabolic responses. Biotechnol Bioeng 89, 556-564.

Mandrand-Berthelot, M. A. \& Lagarde, A. E. (1977). Hit-and-run mechanism for D-glucuronate reduction catalyzed by D-mannonate NAD oxidoreductase of Escherichia coli. Biochim Biophys Acta 483, 623.

Mandrand-Berthelot, M. A., Condemine, G. \& Hugouvieux-CottePattat, N. (2004). Catabolism of hexuronides, hexuronates, aldonates, and aldarates. In EcoSal-Escherichia coli and Salmonella: Cellular And Molecular Biology. Edited by R. Curtis III. Washington, DC: American Society for Microbiology.

Mat-Jan, F., Alam, K. Y. \& Clark, D. P. (1989). Mutants of Escherichia coli deficient in the fermentative lactate dehydrogenase. J Bacteriol 171, 342-348.

Miller, J. H. (1972). Experiments in Molecular Genetics. Cold Spring Harbor, NY: Cold Spring Harbor Laboratory.

Murarka, A., Dharmadi, Y., Yazdani, S. S. \& Gonzalez, R. (2008). Fermentative utilization of glycerol in Escherichia coli and its implications for the production of fuels and chemicals. Appl Environ Microbiol 74, 1124-1135.

Neidhardt, F. C., Bloch, P. L. \& Smith, D. F. (1974). Culture medium for enterobacteria. J Bacteriol 119, 736-747.

Neidhardt, F. C., Ingraham, J. \& Schaechter, M. (1990). Physiology of the Bacterial Cell. Sunderland, MA: Sinauer Associates.

Neidhardt, F. C., Curtiss, R., III, Ingraham, J. L., Lin, E. C. C., Low, K. B., Magasanik, B., Reznikoff, W. S., Riley, M., Schaechter, M. \& Umbarger, H. E. (1996). Escherichia coli and Salmonella: Cellular and Molecular Biology. 2nd edn. Washington, DC: American Society for Microbiology.

Nielsen, J. (2003). It is all about metabolic fluxes. J Bacteriol 185, 7031-7035.

Nielsen, J., Villadsen, J. \& Liden, G. (2003). Biochemical reactions - a first look. In Bioreaction Engineering Principles, pp. 6073. Edited by J. Nielsen, J. Villadsen and G. Lidén. New York: Springer.

Osman, Y. A., Conway, T., Bonetti, S. J. \& Ingram, L. O. (1987). Glycolytic flux in Zymomonas mobilis: enzyme and metabolite levels during batch fermentation. J Bacteriol 169, 3726-3736.

Peekhaus, N. \& Conway, T. (1998). What's for dinner?: EntnerDoudoroff metabolism in Escherichia coli. J Bacteriol 180, 3495-3502.

Pramanik, J. \& Keasling, J. D. (1997). A stoichiometric model of Escherichia coli metabolism: incorporation of growth-rate dependent biomass composition and mechanistic energy requirements. Biotechnol Bioeng 56, 398-421.

Romeo, T. \& Snoep, J. L. (2005). Glycolysis and flux control. In EcoSal-Escherichia coli and Salmonella: Cellular and Molecular Biology. Edited by R. Curtis III. Washington, DC: American Society for Microbiology.

Sambrook, J., Fritsch, E. F. \& Maniatis, T. (1989). Molecular Cloning: a Laboratory Manual, 2nd edn. Cold Spring Harbor, NY: Cold Spring Harbor Laboratory.

Sauer, U., Canonaco, F., Heri, S., Perrenoud, A. \& Fischer, E. (2004). The soluble and membrane-bound transhydrogenases UdhA and PntAB have divergent functions in NADPH metabolism of Escherichia coli. J Biol Chem 279, 6613-6619.

Sawers, R. G. \& Clark, D. P. (2004). Fermentative pyruvate and acetylcoenzyme A metabolism. In EcoSal_Escherichia coli and Salmonella: Cellular and Molecular Biology. Edited by R. Curtis III. Washington, DC: American Society for Microbiology. 
Smith, M. W. \& Neidhardt, F. C. (1983). 2-Oxoacid dehydrogenase complexes of Escherichia coli: cellular amounts and patterns of synthesis. J Bacteriol 156, 81-88.

Snoep, J. L., Teixeira de Mattos, M. J., Postma, P. W. \& Neijssel, O. M. (1990). Involvement of pyruvate dehydrogenase in product formation in pyruvate-limited anaerobic chemostat cultures of Enterococcus faecalis NCTC 775. Arch Microbiol 154, 50-55.

Snoep, J. L., de Graef, M. R., Westphal, A. H., de Kok, A., Teixeira de Mattos, M. J. \& Neijssel, O. M. (1993). Differences in sensitivity to $\mathrm{NADH}$ of purified pyruvate dehydrogenase complexes of Enterococcus faecalis, Lactococcus lactis, Azotobacter vinelandii and Escherichia coli: implications for their activity in vivo. FEMS Microbiol Lett 114, 279283.

Stephanopoulos, G., Aristidou, A. A. \& Nielsen, J. (1998). Metabolic Engineering: Principles and Methodology. San Diego, CA: Academic Press.

Stocchi, V., Cucchiarini, L., Magnani, M., Chiarantini, L., Palma, P. \& Crescentini, G. (1985). Simultaneous extraction and reverse-phase high-performance liquid chromatographic determination of adenine and pyridine nucleotides in human red blood cells. Anal Biochem 146, 118-124.

Stryer, L. (1995). Biochemistry. New York: Freeman.

Tao, H., Gonzalez, R., Martinez, A., Rodriguez, M., Ingram, L. O., Preston, J. F. \& Shanmugam, K. T. (2001). Engineering a homoethanol pathway in Escherichia coli: increased glycolytic flux and levels of expression of glycolytic genes during xylose fermentation. J Bacteriol 183, 2979-2988.

Varenne, S., Casse, F., Chippaux, M. \& Pascal, M. C. (1975). A mutant of Escherichia coli deficient in pyruvate formate lyase. Mol Gen Genet 141, 181-184.

Yazdani, S. S. \& Gonzalez, R. (2008). Engineering Escherichia coli for the efficient conversion of glycerol to ethanol and co-products. Metab Eng 10, 340-351.

Edited by: R. G. Sawers 